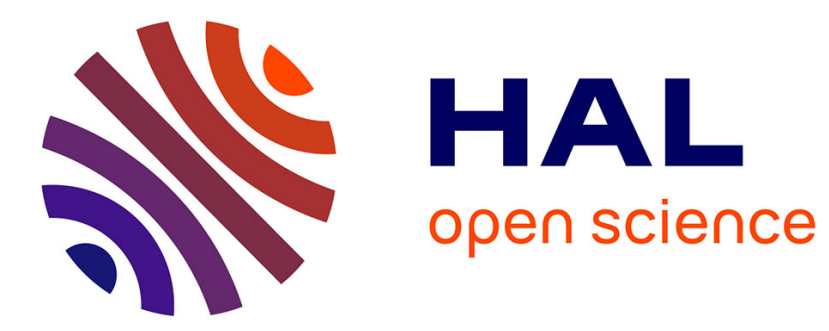

\title{
Optimal control of the atmospheric arc of a space shuttle and numerical simulations with multiple-shooting method
}

Bernard Bonnard, Ludovic Faubourg, Emmanuel Trélat

\section{To cite this version:}

Bernard Bonnard, Ludovic Faubourg, Emmanuel Trélat. Optimal control of the atmospheric arc of a space shuttle and numerical simulations with multiple-shooting method. Mathematical Models and Methods in Applied Sciences, 2005, 15 (1), pp.109-140. hal-00086338

\section{HAL Id: hal-00086338 https://hal.science/hal-00086338}

Submitted on 18 Jul 2006

HAL is a multi-disciplinary open access archive for the deposit and dissemination of scientific research documents, whether they are published or not. The documents may come from teaching and research institutions in France or abroad, or from public or private research centers.
L'archive ouverte pluridisciplinaire HAL, est destinée au dépôt et à la diffusion de documents scientifiques de niveau recherche, publiés ou non, émanant des établissements d'enseignement et de recherche français ou étrangers, des laboratoires publics ou privés. 


\title{
OPTIMAL CONTROL OF THE ATMOSPHERIC ARC OF A SPACE SHUTTLE AND NUMERICAL SIMULATIONS WITH MULTIPLE-SHOOTING METHOD
}

\author{
B. BONNARD, L. FAUBOURG
}

Université de Bourgogne, Département de Mathématiques, LAA0, BP 47870, 21078 Dijon, France.

bbonnard@u-bourgogne.fr, lfaubour@u-bourgogne.fr.

E. TRELAT

Université Paris-Sud, Laboratoire AN-EDP, Mathématiques, UMR 8628, Bâtiment 425, 91405 Orsay, France. emmanuel.trelat@math.u-psud.fr.

\author{
Received (Day Month Year) \\ Revised (Day Month Year) \\ Communicated by (xxxxxxxxxx)
}

\begin{abstract}
This article, continuation of previous works ${ }^{5,3}$, presents the applications of geometric optimal control theory to the analysis of the Earth re-entry problem for a space shuttle where the control is the angle of bank, the cost is the total amount of thermal flux, and the system is subject to state constraints on the thermal flux, the normal acceleration and the dynamic pressure. Our analysis is based on the evaluation of the reachable set using the maximum principle and direct computations with the boundary conditions according to the CNES research project ${ }^{\mathrm{a}}$. The optimal solution is approximated by a concatenation of bang and boundary arcs, and is numerically computed with a multipleshooting method.
\end{abstract}

Keywords: Optimal control with state constraints; control of the atmospheric arc; multiple-shooting method.

AMS Subject Classification: 49K15, 70M20,49M15

\section{Introduction}

\subsection{Presentation of the project}

The project of atmospheric re-entry has been set out by the CNES (french space agency), motivated by the increasing importance of aero-capture techniques in several areas: guidance of aeroassisted orbital transfers; development of reprocessable satellite launchers; problems of re-entry in the atmosphere.

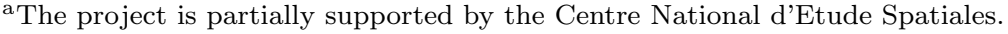


In brief, the atmospheric phase consists in reducing the speed of the engine by friction with the atmosphere, steering the spacecraft from a set of initial points to a prescribed target, taking into account some state-constraints on the thermal flux, on the normal acceleration and on the dynamic pressure. Moreover the aim is to minimize the total thermal flux of the spacecraft. Notice that the main difficulty of the problem is to handle these state constraints; more precisely, since the traversing of the atmosphere is done at very high velocities, the constraint on the thermal flux is predominant. The strategy that has been used by the CNES is due to Harpold and Graves ${ }^{10}$, and consists in tracking boundary arcs, see Section 1.4.

More specifically, this project is part of the famous project Mars Sample Return developped by the CNES. This article is the conclusion of the study by the authors of the optimization problem. The stabilization part has been investigated by another group $^{8}$. In particular, specific data were imposed by the CNES: initial and final conditions (see Table 1), constraints on the state, specific numerical data concerning the shuttle, given in Appendix.

Our control is the aerodynamic configuration of the shuttle. It happens that, if the altitude is between about $20 \mathrm{~km}$ and $120 \mathrm{~km}$, the aerodynamic forces can be employed in order to slow down the spacecraft, itself behaving like a glider, that is, there is no thrust.

More precisely the model is as follows.

\subsection{The model}

Let 0 be the center of the planet, $K=N S$ be the axis of rotation of the Earth and $\vec{\Omega}$ be the angular velocity oriented along $K$ and with constant modulus $\Omega$. We note $E=\left(e_{1}, e_{2}, e_{3}\right), e_{3}=K$ an inertial frame centered at 0 . The reference frame is the quasi-inertial frame $R_{1}=(I, J, K)$ with origin 0 rotating around $K$ with angular speed $\Omega$, and $I$ is chosen to intersect the Greenwich meridian. Let $r_{T}$ denote the Earth radius and let $G$ be the mass center of the shuttle. The spherical coordinates of $G$ are denoted $(r, l, L), r$ being the distance $O G, h=r-r_{T}$ is the altitude, $l$ the longitude and $L$ is the latitude. We denote $R_{1}^{\prime}=\left(e_{r}, e_{l}, e_{L}\right)$ a moving frame centered at $G$, where $e_{r}$ is the local vertical direction, $\left(e_{l}, e_{L}\right)$ is the local horizontal plane and $e_{L}$ is pointing to the north.

Let $\xi: t \mapsto(x(t), y(t), z(t))$ be the trajectory of $G$ measured in the quasi-inertial frame attached to the planet and let $\vec{v}=\dot{x} I+\dot{y} J+\dot{z} K$ be the relative velocity. It is convenient in our problem to parametrize $v$ by the modulus and two angles: the path inclination $\gamma$ which is the angle of $\vec{v}$ with respect to the horizontal plane; the azimuth angle $\chi$ which is the angle of the projection of $\vec{v}$ onto the horizontal plane with respect to the axis $e_{L}$. We denote $(i, j, k)$ the orthonormal frame defined by $i=\vec{v} / v, j$ is the unitary vector in the plane $\left(i, e_{r}\right)$ perpendicular to $i$ and oriented by $j . e_{r}>0$ and $k=i \wedge j$.

The system is written in the coordinates $(r, v, \gamma, L, l, \chi)$. The forces acting on the vehicle are the gravitational force $\vec{P}=m \vec{g}$, and the aerodynamic force, which 
consists of a drag force $\vec{D}=\left(\frac{1}{2} \rho S C_{D} v^{2}\right) i$, opposite to $\vec{v}$, and of a lift force $\vec{L}=$ $\left(\frac{1}{2} \rho S C_{L} v^{2}\right)(j \cos \mu+k \sin \mu)$, perpendicular to $\vec{v}$, where $\mu$ is the angle of bank, $\rho=\rho(r)$ is the air density, $S$ is the reference area and $C_{D}, C_{L}$ are respectively the lift and drag coefficients depending on the angle of attack $\alpha$ of the vehicle and the Mach number. The coefficients $C_{D}$ and $C_{L}$ are tabulated and their values are given in Section 4. Concerning the air density we take an exponential model $\rho(r)=\rho_{0} \exp \left(-\left(r-r_{T}\right) / h_{s}\right)$. Due to the choice of a non inertial frame to represent the system, the spacecraft is submitted to the Coriolis force $2 m \vec{\Omega} \wedge \dot{q}$ and to the centripetal force $m \vec{\Omega} \wedge(\vec{\Omega} \wedge q$ ), where $q$ (resp. $\dot{q}$ ) denotes the relative position (resp. relative velocity) of the spacecraft.

During the atmospheric arc the shuttle behaves as a glider. The physical control is the lift force $\vec{L}$ whose orientation is represented by the angle of bank $\mu(t) \in \mathbb{R}$ which can be adjusted.

Remark 1.1. If we set $u_{1}=\cos \mu$ and $u_{2}=\sin \mu$, then $u_{1}$ is a direct control on the flight path angle $\gamma$, and $u_{2}$ is a control on the azimuth $\chi$. Moreover the sign of $u_{2}$ allows the spacecraft to turn left or right ${ }^{7}$.

The model ${ }^{16,7}$ is a nonlinear control system in dimension 6 , controlled by the angle of bank $\mu$. The equations of the system are

$$
\begin{aligned}
\frac{d r}{d t}= & v \sin \gamma \\
\frac{d v}{d t}= & -g \sin \gamma-\frac{1}{2} \rho \frac{S C_{D}}{m} v^{2}+\Omega^{2} r \cos L(\sin \gamma \cos L-\cos \gamma \sin L \cos \chi) \\
\frac{d \gamma}{d t}= & \cos \gamma\left(-\frac{g}{v}+\frac{v}{r}\right)+\frac{1}{2} \rho \frac{S C_{L}}{m} v \cos \mu \\
& \quad+2 \Omega \cos L \sin \chi+\Omega^{2} \frac{r}{v} \cos L(\cos \gamma \cos L+\sin \gamma \sin L \cos \chi) \\
\frac{d L}{d t}= & \frac{v}{r} \cos \gamma \cos \chi \\
\frac{d l}{d t}= & \frac{v}{r} \frac{\cos \gamma \sin \chi}{\cos L} \\
\frac{d \chi}{d t}= & \frac{1}{2} \rho \frac{S C_{L}}{m} \sin \mu \frac{v}{\cos \gamma}+\frac{v}{r} \cos \gamma \tan L \sin \chi+2 \Omega(\sin L-\tan \gamma \cos L \cos \chi(1.7) \\
& \quad+\Omega^{2} \frac{r}{v} \frac{\sin L \cos L \sin \chi}{\cos \gamma}
\end{aligned}
$$

\subsection{Optimal control problems}

The problem is to steer the vehicle from an initial manifold $M_{0}$ to a terminal manifold $M_{1}$, the transfer time $t_{f}$ is free and the boundary conditions are given in Table 1 according to the CNES research project. Observe that we have two distinct problems depending on whether the initial longitude is fixed or free. There are state constraints of the form $c_{i}(q) \leq 0$ for $i=1,2,3$, which consist of a constraint on the thermal flux $\varphi=C_{q} \sqrt{\rho} v^{3} \leq \varphi^{\max }$, where $C_{q}$ is a constant; a constraint on the 


\begin{tabular}{|l|c|c|}
\hline & Initial conditions & Terminal conditions \\
\hline altitude $h$ & $119.82 \mathrm{~km}$ & $15 \mathrm{~km}$ \\
\hline velocity $v$ & $7404.95 \mathrm{m.s}^{-1}$ & $445 \mathrm{m.s}^{-1}$ \\
\hline flight angle $\gamma$ & $-1.84 \mathrm{deg}$ & free \\
\hline latitude $L$ & 0 & $10.99 \mathrm{deg}$ \\
\hline longitude $l$ & free or fixed to $116.59 \mathrm{deg}$ & $166.48 \mathrm{deg}$ \\
\hline azimuth $\chi$ & free & free \\
\hline
\end{tabular}

Table 1. Boundary conditions

normal acceleration $\gamma_{n}=\gamma_{n_{0}} \rho v^{2} \leq \gamma_{n}^{\max }$, where $\gamma_{n_{0}}$ is a constant; a constraint on the dynamic pressure $\frac{1}{2} \rho v^{2} \leq P^{\max }$. They are represented on Fig. 1, in the flight domain, in terms of the modulus of the drag force $d=\frac{1}{2} S C_{D} \rho v^{2}$, and the modulus of the velocity.

$d$

Fig. 1. Constraints - Harpold and Graves strategy

The optimal control problem is to minimize the total amount of thermal flux $J(\mu)=\int_{0}^{t_{f}} C_{q} \sqrt{\rho} v^{3} d t$. Notice that if we introduce the new time parameter $d s=\varphi d t$, our problem reduces to a time minimal problem.

\subsection{Harpold and Graves strategy}

If we approximate the system by $\dot{v}=-d / m$, the cost can be written as $J(\mu)=$ $K \int_{v_{f}}^{v_{0}} \frac{v^{2}}{\sqrt{d}} d v$, where $K>0$, and the optimal policy consists in maximizing the drag term $d$ during the flight. This is the strategy used by ${ }^{10}$, which reduces the problem to find a trajectory of the system tracking the boundary of the domain in the following order: thermal flux, normal acceleration, dynamic pressure, see Fig. 1. However the trajectory obtained by this pioneering method is not optimal because of the approximation. 


\subsection{Properties and structure of the system}

Reparametrizing by the flux, the problem is to minimize the time $s$ for a system of the form $q^{\prime}(s)=X(q(s))+u_{1}(s) Y_{1}(q(s))+u_{2}(s) Y_{2}(q(s))$, where $u_{1}=\cos \mu$, $u_{2}=\sin \mu$ and $q=(r, v, \gamma, L, l, \chi)$. If we split the coordinates into $q_{1}=(r, v, \gamma)$ and $q_{2}=(L, l, \chi)$, the system can be decomposed into

$$
\dot{q}_{1}=f_{1}\left(q_{1}, u_{1}\right)+O(\Omega), \dot{q}_{2}=f_{2}\left(q, u_{2}\right)+O(\Omega) .
$$

If we neglect the rotation $\Omega$ of the planet, the first subsystem governing the longitudinal motion is independent of $q_{2}$ and is given by

$$
\begin{aligned}
& \frac{d r}{d t}=(v \sin \gamma) \psi, \quad \frac{d v}{d t}=-\left(g \sin \gamma+\frac{1}{2} \rho \frac{S C_{D}}{m} v^{2}\right) \psi, \\
& \frac{d \gamma}{d t}=\left(\cos \gamma\left(-\frac{g}{v}+\frac{v}{r}\right)+\frac{1}{2} \rho \frac{S C_{L}}{m} v \cos \mu\right) \psi,
\end{aligned}
$$

and the second system governing the lateral motion is

$$
\begin{aligned}
\frac{d L}{d t} & =\left(\frac{v}{r} \cos \gamma \cos \chi\right) \psi, \quad \frac{d l}{d t}=\left(\frac{v}{r} \frac{\cos \gamma \sin \chi}{\cos L}\right) \psi, \\
\frac{d \chi}{d t} & =\left(\frac{1}{2} \rho \frac{S C_{L}}{m} \sin \mu \frac{v}{\cos \gamma}+\frac{v}{r} \cos \gamma \tan L \sin \chi\right) \psi,
\end{aligned}
$$

where $\psi=1 / \varphi$. The flight duration is about 1000 seconds and the coupling of the longitudinal motion with the lateral motion via $\Omega$ is small.

\subsection{Kepler motion}

If we neglect the aerodynamic force and if $g=g_{0} / r^{2}$, then the motion is a Kepler planar motion. In the inertial frame, the trajectories are conics and ellipses if the energy $E$ is strictly negative. In our coordinate system, the Kepler motion can be seen by taking $L=0, \chi=\pi / 2$ (this normalizes the plane of the motion to the equatorial plane). The drag force is contained in the plane, and the same property holds for the lift force if $\sin \mu=0$, the effect of $\sin \mu$ being to turn left or right and change the inclination of the osculating plane. Also the Coriolis force is oriented along $\vec{\Omega} \wedge \dot{q}$ where $\vec{\Omega}$ is perpendicular to the equator. Hence we introduce the following controlled Kepler equations with dissipative force

$$
\begin{aligned}
& \frac{d r}{d t}=v \sin \gamma, \quad \frac{d v}{d t}=-g \sin \gamma-\frac{1}{2} \rho \frac{S C_{D}}{m} v^{2}+\Omega^{2} r \sin \gamma \\
& \frac{d \gamma}{d t}=\cos \gamma\left(-\frac{g}{v}+\frac{v}{r}\right)+\frac{1}{2} \rho \frac{S C_{L}}{m} v \cos \mu+2 \Omega+\Omega^{2} \frac{r}{v} \cos \gamma, \quad \frac{d l}{d t}=\frac{v}{r} \cos \gamma(.1
\end{aligned}
$$

If one does not take into account the state constraints, this system actually reduces to a system in dimension 3 , representing the longitudinal motion $(r, v, \gamma)$ if the longitude $l$ is not controlled. If we take into account the state constraints, a control to track the boundary is not of the form $\cos \mu= \pm 1$ and the latitude is modified. 
Notice also that the equatorial plane is fixed by the axis $N S$ of rotation. But if $\Omega=0$, the axis $N S$ and the invariant plane can be chosen arbitrarily, where the system reduces to (1.13) if $\cos \mu= \pm 1$. This geometric property can be expressed in proper coordinates where the azimuth $\chi$ is replaced by the inclination of the osculating plane with respect to a reference plane, e.g. the equatorial plane.

\section{Reachable set and maximum principle}

\subsection{Optimal control without state constraint}

We first analyze the problem without state constraint. Consider a system of the form

$$
\dot{q}(t)=f(q(t), u(t))
$$

where $q(t) \in \mathbb{R}^{\mathrm{n}}, u(t) \in U \subset \mathbb{R}^{\mathrm{p}}, f$ is a smooth mapping and the cost to be minimized is of the form $C(u)=\int_{0}^{T} f^{0}(q, u) d t$, where $f^{0}$ is a smooth mapping, and the transfer time $T$ is not fixed. The class of admissible controls is the set $\mathcal{U}$ of measurable bounded mappings $u:[0, T] \rightarrow U$. We denote by $q\left(t, q_{0}, u\right)$ the solution of (2.1) associated to $u$ and defined on a subinterval of $[0, T]$. The system (2.1) can be extended in $\mathbb{R}^{\mathrm{n}+1}$ by

$$
\dot{q}=f(q, u), \dot{q}^{0}=f^{0}(q, u),
$$

with $q^{0}(0)=0$, and $q^{0}(T)$ represents the cost. We denote by $\tilde{q}\left(t, q_{0}, u\right)$ the corresponding trajectory. If $q_{0}$ is fixed, let us denote respectively $R\left(q_{0}, T\right)$ and $\tilde{R}\left(q_{0}, T\right)$ the reachable sets at time $T$ corresponding to (2.1) and (2.2), and $R\left(q_{0}\right), \tilde{R}\left(q_{0}\right)$ the reachable sets. Fix $q_{0}, T$, and consider the end-points mappings $E^{q_{0}, T}: u(.) \mapsto$ $q\left(T, q_{0}, u\right)$, and $\tilde{E}^{q_{0}, T}: u(.) \mapsto \tilde{q}\left(T, q_{0}, u\right)$. Then the reachable sets at time $T$ are the images of the set $\mathcal{U}$ by the end-points mappings.

Maximum principle. Consider the optimal control problem with boundary conditions $q_{0}=q(0) \in M_{0}, q_{1}=q(T) \in M_{1}$, where $M_{0}, M_{1}$ are smooth sub-manifolds in $\mathbb{R}^{\mathrm{n}}$ and the transfer time $T$ is not fixed. If $u$ is optimal with response defined on $[0, T]$, and shortly denoted $q(t)$, then there exists $\tilde{p}(t)=\left(p(t), p^{0}(t)\right) \in\left(\mathbb{R}^{\mathrm{n}} \times \mathbb{R}\right) \backslash\{0\}$ absolutely continuous on $[0, T]$ such that there holds a.e. on $[0, T]$

$$
\dot{q}=\frac{\partial \tilde{H}}{\partial p}, \dot{p}=-\frac{\partial \tilde{H}}{\partial q}, \text { and } \tilde{H}(q, p, u)=\max _{v \in U} \tilde{H}(q, p, v)=0,
$$

where $\tilde{H}=\langle p, f(q, u)\rangle+p^{0} f^{0}(q, u)$ is the Hamiltonian of the system, $p^{0}$ is a nonpositive real number, and $p$ satisfies the transversality conditions $p(0) \perp T_{q_{0}} M_{0}$, and $p(T) \perp T_{q_{1}} M_{1}$, where $T_{q_{i}} M_{i}$ are the tangent spaces ${ }^{17}$.

Definition 2.1. A triple $(q(\cdot), p(\cdot), u(\cdot))$ solution of the maximum principle is called an extremal, and $\left(p(\cdot), p^{0}\right)$ is called adjoint vector. An extremal is said normal if $p^{0} \neq 0$ and abnormal if $p^{0}=0$. 
Computation of extremals. Reparametrizing by the flux, the problem reduces to a time minimal control problem. The system is of the form $q^{\prime}(s)=f(q(s), \mu(s))=$ $X(q(s))+u_{1}(s) Y_{1}(q(s))+u_{2}(s) Y_{2}(q(s))$, where $q=(r, v, \gamma, L, l, \chi) \in \mathbb{R}^{6}, u_{1}=\cos \mu$, and $u_{2}=\sin \mu$. The Hamiltonian writes $H=\langle p, X\rangle+u_{1}\left\langle p, Y_{1}\right\rangle+u_{2}\left\langle p, Y_{2}\right\rangle$. Since $\mu \in$ $\mathbb{R}$, the maximization condition leads to $\partial H / \partial \mu=-\sin \mu\left\langle p, Y_{1}\right\rangle+\cos \mu\left\langle p, Y_{2}\right\rangle=0$, and moreover the solution has to satisfy the Legendre condition $\partial^{2} H / \partial \mu^{2} \leq 0$. If the strong Legendre condition, namely $\partial^{2} H / \partial u^{2}<0$, is satisfied, the extremal controls can be computed locally as $(q, p) \mapsto \hat{u}(q, p)$ using the implicit function theorem and the extremals are smooth. This is a general method, but in our case a simple parameterization is obtained from the maximum principle by maximizing the Hamiltonian $H$ over the circle $u_{1}^{2}+u_{2}^{2}=1$, and hence

$$
u_{1}=\left\langle p, Y_{1}\right\rangle / \sqrt{\left\langle p, Y_{1}\right\rangle^{2}+\left\langle p, Y_{2}\right\rangle^{2}}, u_{2}=\left\langle p, Y_{2}\right\rangle / \sqrt{\left\langle p, Y_{1}\right\rangle^{2}+\left\langle p, Y_{2}\right\rangle^{2}},
$$

outside the set where $\left\langle p, Y_{1}\right\rangle=\left\langle p, Y_{2}\right\rangle=0$. The problem is not convex because the control domain is $u_{1}^{2}+u_{2}^{2}=1$ and its relaxation is obtained by considering the control domain $u_{1}^{2}+u_{2}^{2} \leq 1$. This extends the set of extremals of our original problem.

Definition 2.2. The surface $\Sigma:\left\langle p, Y_{1}\right\rangle=\left\langle p, Y_{2}\right\rangle=0$, is called the switching surface. The mapping $\Phi(t)=\left(\Phi_{1}(t), \Phi_{2}(t)\right), \Phi_{i}=\left\langle p, Y_{i}(q)\right\rangle$ evaluated along an extremal is called the switching mapping. Extremals not intersecting $\Sigma$ are said of order 0 , and are smooth, the control being given by (2.3). Extremals of the relaxed problem such that $\Phi \equiv 0$ are said singular.

First- and second-order variation of the end-point mapping. If we endow the set of controls with the $L^{\infty}$-topology, it is well-known that the end-point mapping is smooth and the successive derivatives can be computed as follows. Take a reference control $u \in L^{\infty}[0, T]$ and assume that the corresponding trajectory, denoted $q(t)$, is defined on the whole $[0, T]$. Then the end-point mapping with $q(0)=q_{0}$ is defined on a neighborhood $V$ of $u$. Let $q(t)+\xi(t)$ be the trajectory associated to $u+v \in V$. Since $f$ is smooth,

$$
\begin{aligned}
f(q+\xi, u+v)= & f(q, u)+\frac{\partial f}{\partial q}(q, u) \xi+\frac{\partial f}{\partial u}(q, u) v \\
& +\frac{\partial^{2} f}{\partial q \partial u}(q, u)(\xi, v)+\frac{1}{2} \frac{\partial^{2} f}{\partial q^{2}}(q, u)(\xi, \xi)+\frac{1}{2} \frac{\partial^{2} f}{\partial u^{2}}(q, u)(v, v)+\ldots
\end{aligned}
$$

Moreover $\dot{q}+\dot{\xi}=f(q+\xi, u+v)$, and $\xi$ can be written as $\delta_{1} q+\delta_{2} q+\ldots$, where $\delta_{1} q$ is linear in $v, \delta_{2} q$ is quadratic in $v$, etc, and by identification one gets

$$
\begin{gathered}
\dot{\delta_{1} q}=\frac{\partial f}{\partial q}(q, u) \delta_{1} q+\frac{\partial f}{\partial u}(q, u) v \\
\dot{\delta_{2} q}=\frac{\partial f}{\partial q}(q, u) \delta_{2} q+\frac{\partial^{2} f}{\partial q \partial u}(q, u)\left(\delta_{1} q, v\right)+\frac{1}{2} \frac{\partial^{2} f}{\partial q^{2}}(q, u)\left(\delta_{1} q, \delta_{1} q\right)+\frac{1}{2} \frac{\partial^{2} f}{\partial u^{2}}(q, u)(v, v),(2.5)
\end{gathered}
$$

where $\delta_{1} q(0)=\delta_{2} q(0)=0$. Hence $\delta_{1} q$ and $\delta_{2} q$ can be computed integrating linear differential equations. 
Lemma 2.1. The Fréchet derivative $E^{\prime q_{0}, T}$ of the end-point mapping is $\delta_{1} q(T)$.

Definition 2.3. A control $u(t), 0 \leq t \leq T$, with trajectory defined on $[0, T]$, is said to be singular if the end-point mapping is singular at $u$, i.e. the Fréchet derivative of the end-point mapping is not surjective at $u$. For each $t \in(0, T]$, the restriction of $u$ on $[0, t]$ is singular. Denote $K(t)$ the image of $L^{\infty}(0, t)$ by the Fréchet derivative of $E^{q_{0}, t}$ evaluated at $u$ restricted to $[0, t]$. By construction $K(t)$ is a vector space of codimension $k(t) \geq 1$, called codimension of the singularity.

The following result is standard.

Proposition 2.1. Let $u(t), 0 \leq t \leq T$, be a singular control and let $q(t)$ be the associated trajectory defined on $[0, T]$. Then there exists $p(t) \in \mathbb{R}^{\mathrm{n}} \backslash\{0\}$, such that there holds a.e. on $[0, T]$

$$
\dot{q}=\frac{\partial H}{\partial p}, \dot{p}=-\frac{\partial H}{\partial q}, \frac{\partial H}{\partial u}=0,
$$

where $H(q, p, u)=\langle p, f(q, u)\rangle$. Moreover $p(t) \perp K(t)$ for $0<t \leq T$.

Definition 2.4. Let $u$ be a singular control with response $q(t)$ defined on $[0, T]$. Let $N \subset L^{\infty}(0, T)$ be the kernel of $E^{\prime}$ evaluated at $u$ and let $p \in \mathbb{R}^{\mathrm{n}} \backslash\{0\}$ be an adjoint vector such that $\left\langle p, E_{u}^{\prime}(v)\right\rangle=0, \forall v \in L^{\infty}$. The intrinsic second-order derivative associated to $(q, p, u)$ is the mapping $E^{\prime \prime}: v \in N \mapsto\left\langle p, \delta_{2} q\right\rangle$, where $\delta_{2} q$ is the second variation defined by $(2.5)$.

Remark 2.1. If the codimension of the singularity is one, $p$ is unique up to a scalar. In the general case ${ }^{1}$, along a singular trajectory we have a family of intrinsic second-order derivatives parametrized by $p$.

Application to the space shuttle. Extremals of the (non convex) problem project onto singular trajectories for the single input system $\dot{q}=f(q, \mu)$, where the control is the angle of bank $\mu$. Singular controls of the relaxed problem correspond to singular trajectories of the bi-input system $\dot{q}=X(q)+u_{1} Y_{1}(q)+u_{2} Y_{2}(q)$; they can be computed ${ }^{5}$ using Lie brackets. Singular trajectories are feedback-invariant ${ }^{2}$ and for their computation we may replace $Y_{1}=\frac{1}{2} \rho \frac{S C_{L}}{m} v \frac{\partial}{\partial \gamma}$ by the constant vector field $\partial / \partial \gamma$, and $Y_{2}=\frac{1}{2} \rho \frac{S C_{L}}{m} \frac{v}{\cos \gamma} \frac{\partial}{\partial \chi}$ by $\partial / \partial \chi$. Then there holds $\left[Y_{1}, Y_{2}\right]=0$ and we can consider $\gamma$ and $\chi$ as direct controls. Singular trajectories are the singularities of the corresponding end-point mapping.

Necessary and sufficient conditions of optimality for the $L^{\infty}$-topology in the smooth case. The aim is to briefly recall recent works developed in ${ }^{4}$ and ${ }^{18}$ to get necessary and sufficient conditions of optimality in the $L^{\infty}$-topology and to give an algorithm. This theory is based on the intrinsic second-order derivative and on the concept of conjugate time.

Definition 2.5. Consider the time minimal control problem for the control system $\dot{q}=f(q, u)$ where $u \in \mathbb{R}$. Let $u(t), t \in[0, T]$, be a smooth extremal control with 
response defined on $[0, T]$. The first conjugate time $t_{1 c}$ is characterized by the fact that $u$ is locally optimal on $[0, t]$ if $t<t_{1 c}$ and no more optimal if $t>t_{1 c}$, for the $L^{\infty}$-topology.

Let $q(\cdot)$ be a one-to-one reference trajectory, and let $(q(\cdot), p(\cdot))$ be an extremal lift of the trajectory. We assume that $\left(H_{1}\right)$ the system is real analytic, $\left(H_{2}\right)$ the codimension of the singularity is one, $\left(H_{3}\right)$ the strong Legendre condition $\partial^{2} H / \partial u^{2}<0$ is satisfied, $\left(H_{4}\right)$ the reference extremal is normal. Using the implicit function theorem and $\left(H_{3}\right)$, the extremal control can be locally computed as a feedback $\hat{u}(q, p)$ solving $\partial H / \partial u=0$. Let $\hat{H}(q, p)=H(q, p, \hat{u}(q, p))$. Then under the previous assumptions our reference extremal is embedded into a family of extremals solutions of the Hamiltonian differential equation associated to $\hat{H}$.

Let $q\left(t, q_{0}, t_{0}\right), p\left(t, q_{0}, p_{0}\right)$ denote such an extremal starting at time $t=0$ from $\left(q_{0}, p_{0}\right)$. By homogeneity, we can assume that $p_{0}$ belongs to $\mathbb{P}^{n-1}$, the projective space in $\mathbb{R}^{n}$, and from $\left(H_{4}\right), \hat{H}>0$. The exponential mapping $\exp _{q_{0}}: \mathbb{R}_{+} \times \mathbb{P}^{n-1} \rightarrow$ $\mathbb{R}^{\mathrm{n}}$ is defined by $\exp _{q_{0}}\left(t, p_{0}\right)=q\left(t, q_{0}, p_{0}\right)$.

Definition 2.6. Let $z=(q, p)$. We call variational system associated to $\hat{H}$ along the reference extremal the system $\dot{\delta} z=\frac{\partial \hat{H}}{\partial z}(z(t)) \delta z$. A vertical Jacobi field $J(t)=$ $(\delta q(t), \delta p(t))$ on $[0, T]$ is a nontrivial solution of the variational system such that $\delta q(0)=0$.

Proposition 2.2. ${ }^{18,4}$ Under the previous assumptions, the first conjugate time $t_{1 c}$ is the first $t$ so that one of the following equivalent conditions holds:

(1) There exists a vertical Jacobi field $J(\cdot)=(\delta q(\cdot), \delta p(\cdot))$ on $[0, T]$ satisfying $\delta q(t)=0$.

(2) The intrinsic second-order derivative $E^{\prime \prime}$ has a nontrivial kernel at $t$.

(3) The exponential mapping $\exp _{q_{0}}$ is not submersive at $\left(t, p_{0}\right)$.

The algorithm. Conjugate times are computed along extremals of order 0 under generic assumptions. Let us consider a reference extremal $z(t)=(q(t), p(t)), t \in$ $[0, T]$, associated to the control $\mu$ and to the initial adjoint vector $p_{0}$. We suppose that assumptions $\left(H_{1}-H_{4}\right)$ are satisfied. Then the first conjugate time $t_{1 c}$ is the first $t$ at which $C(t)=\operatorname{det}\left(\delta q_{1}(t), \ldots, \delta q_{n-1}(t), f(q(t), \mu(t))\right)$ vanishes, where $J_{i}=\left(\delta q_{i}, \delta p_{i}\right)$ is the vertical Jacobi field with initial condition $\delta p_{i}(0)$, and where $\left(\delta p_{1}(0), \ldots, \delta p_{n-1}(0)\right)$ is a basis of the tangent space to $\mathbb{P}^{n-1}$ at $p_{0}$.

Remark 2.2. Here we consider an optimal control problem with fixed extremities but the result can be straightforwardly extended to the case of initial and terminal manifolds and leads to the concept of focal points, up to changing the boundary conditions for the variational system.

Remark 2.3. Proposition 2.2 relates the computation of conjugate times to a spectral property of the intrinsic second-order derivative and to a property of the extremal flow. If the reference extremal is not smooth, conjugate times can be still 
computed in $L^{1}$-topology using the extremal flow and the theory of envelopes ${ }^{20}$ for broken extremals.

\subsection{Optimal control with state constraints}

Consider the time minimal control problem for a system of the form $\dot{q}=f(q, u)$, $u \in U \subset \mathbb{R}$, with a scalar state constraint of the form $c(q) \leq 0$.

Definition 2.7. The generic order of the constraint is the first integer $m$ such that the control $u$ appears explicitly in the $m$-order time derivative of $t \mapsto c(q(t))$ where $q$ is a trajectory of the system. A boundary arc $t \mapsto q_{b}(t)$ with boundary control $u_{b}$ is an arc not reduced to a point and contained in $c=0$. If the order of the constraint is $m$, a boundary arc and the associated control can be generically computed by differentiating $m$ times the mapping $t \mapsto c(q(t))$. A boundary arc is contained in $c=\dot{c}=\ldots=c^{(m-1)}=0$; the constraint $c=0$ is called primary and the constraints $\dot{c}=\ldots=c^{(m-1)}=0$ are called secondary.

Maximum principle. A general maximum principle has been proved in the constrained case, see for instance ${ }^{12}$. We recall here a stronger version ${ }^{13,15}$. Let $q(\cdot)$ be a piecewise smooth solution meeting the boundary of the domain with order equal to the generic order of the constraint. Define the Hamiltonian as $H(q, p, u, \eta)=\langle p, f(q, u)\rangle+\eta c(q)$, where $p$ is the adjoint vector and $\eta$ is the Lagrange multiplier of the constraint. If $q(\cdot)$ is optimal then it satisfies the necessary optimality conditions

$$
\dot{q}=\frac{\partial H}{\partial p}, \dot{p}=-\frac{\partial H}{\partial q}, \text { and } H(q, p, u, \eta)=\max _{v \in U} H(q, p, v, \eta),
$$

where $t \mapsto \eta(t)$ is continuous along the boundary arcs and satisfies $\eta(t) c(q(t))=0$. At a contact or a junction time $t_{i}$ there holds $H\left(t_{i}+\right)=H\left(t_{i}-\right)$, and $p\left(t_{i}+\right)=$ $p\left(t_{i}-\right)+\nu_{i} \partial c / \partial q$,for $\nu_{i} \geq 0$. Moreover the transversality conditions still hold at the extremities.

\section{The space shuttle re-entry problem}

The first part of this section is devoted to compute extremals of the system without taking into account the state constraints; our algorithm to compute conjugate times is applied along an extremal starting from our initial conditions. Then boundary controls and boundary arcs are calculated for the constraints on the thermal flux and on the normal acceleration, which happen to be the only active constraints in our problem; the explicit computations are of course necessary for numerical simulations but can be skipped by the reader. We then present the key point of this section, namely the analysis of the longitudinal and lateral motion. It is applied to compute an approximation of the optimal solutions. 


\subsection{Extremal system for the problem without state constraint}

The computations are quite intricate and were realized using formal computations in Maple. For numerical reasons, the trajectories are not reparametrized using the flux and the Hamiltonian is $\tilde{H}=\left\langle p, X+u_{1} Y_{1}+u_{2} Y_{2}\right\rangle+p^{0} \varphi$ where $p^{0} \leq 0$. According to (2.3), an extremal control is given outside the switching surface, $p_{\gamma}=p_{\chi}=0$ by

$$
u_{1}=\cos \mu=\frac{\cos \gamma p_{\gamma}}{\sqrt{\cos ^{2} \gamma p_{\gamma}^{2}+p_{\chi}^{2}}}, u_{2}=\sin \mu=\frac{p_{\chi}}{\sqrt{\cos ^{2} \gamma p_{\gamma}^{2}+p_{\chi}^{2}}} .
$$

The extremal system is the following.

$$
\begin{gathered}
\frac{d r}{d t}=v \sin \gamma \\
\frac{d v}{d t}=-\frac{g_{0} \sin \gamma}{r^{2}}-k \rho v^{2}+\Omega^{2} r \cos L(\sin \gamma \cos L-\cos \gamma \sin L \cos \chi) \\
\frac{d \gamma}{d t}=\left(-\frac{g_{0}}{r^{2} v}+\frac{v}{r}\right) \cos \gamma+k^{\prime} \rho v \cos \mu+2 \Omega \cos L \sin \chi \\
\quad+\frac{\Omega^{2} r}{v} \cos L(\cos \gamma \cos L+\sin \gamma \sin L \cos \chi)
\end{gathered}
$$

$$
\begin{aligned}
\frac{d L}{d t}= & \frac{v}{r} \cos \gamma \cos \chi \\
\frac{d l}{d t}= & \frac{v}{r} \frac{\cos \gamma \sin \chi}{\cos L} \\
\frac{d \chi}{d t}= & \frac{k^{\prime} \rho v}{\cos \gamma} \sin \mu+\frac{v}{r} \cos \gamma \tan L \sin \chi+2 \Omega(\sin L-\tan \gamma \cos L \cos \chi) \\
& +\Omega^{2} \frac{r}{v} \frac{\sin L \cos L \sin \chi}{\cos \gamma}
\end{aligned}
$$

$$
\begin{aligned}
\frac{d p_{r}}{d t}= & -p_{v}\left(2 \frac{g_{0} \sin \gamma}{r^{3}}+\frac{k \rho v^{2}}{h_{s}}+\Omega^{2} \cos L(\sin \gamma \cos L-\cos \gamma \sin L \cos \chi)\right) \\
& -p_{\gamma}\left(\left(2 \frac{g_{0}}{r^{3} v}-\frac{v}{r^{2}}\right) \cos \gamma-\frac{k^{\prime} \rho v}{h_{s}} \cos \mu+\frac{\Omega^{2}}{v} \cos L(\cos \gamma \cos L+\sin \gamma \sin L \cos \chi)\right) \\
& +p_{L} \frac{v}{r^{2}} \cos \gamma \cos \chi+p_{l} \frac{v}{r^{2}} \frac{\cos \gamma \sin \chi}{\cos L} \\
& -p_{\chi}\left(-\frac{k^{\prime} \rho v}{h_{s} \cos \gamma} \sin \mu-\frac{v}{r^{2}} \cos \gamma \tan L \sin \chi+\frac{\Omega^{2}}{v} \frac{\sin L \cos L \sin \chi}{\cos \gamma}\right) \\
& +p^{0} \frac{C_{q} \sqrt{\rho} v^{3}}{2 h_{s}}
\end{aligned}
$$

$$
\frac{d p_{v}}{d t}=-p_{r} \sin \gamma+2 p_{v} k \rho v-p_{\gamma}\left(\left(\frac{g_{0}}{r^{2} v^{2}}+\frac{1}{r}\right) \cos \gamma+k^{\prime} \rho \cos \mu\right.
$$




$$
\begin{aligned}
& \left.-\frac{\Omega^{2} r}{v^{2}} \cos L(\cos \gamma \cos L+\sin \gamma \sin L \cos \chi)\right)-p_{L} \frac{\cos \gamma \cos \chi}{r}-p_{l} \frac{\cos \gamma \sin \chi}{r \cos L} \\
& -p_{\chi}\left(\frac{k^{\prime} \rho}{\cos \gamma} \sin \mu+\frac{\cos \gamma \tan L \sin \chi}{r}-\frac{\Omega^{2} r}{v^{2}} \frac{\sin L \cos L \sin \chi}{\cos \gamma}\right) \\
& -3 p^{0} C_{q} \sqrt{\rho} v^{2}
\end{aligned}
$$

$$
\begin{aligned}
\frac{d p_{\gamma}}{d t}= & -p_{r} v \cos \gamma-p_{v}\left(-\frac{g_{0}}{r^{2}} \cos \gamma+\Omega^{2} r \cos L(\cos \gamma \cos L+\sin \gamma \sin L \cos \chi)\right) \\
& -p_{\gamma}\left(\left(\frac{g_{0}}{r^{2} v}-\frac{v}{r}\right) \sin \gamma+\frac{\Omega^{2} r}{v} \cos L(-\sin \gamma \cos L+\cos \gamma \sin L \cos \chi)\right) \\
& +p_{L} \frac{v}{r} \sin \gamma \cos \chi+p_{l} \frac{v}{r} \frac{\sin \gamma \sin \chi}{\cos L} \\
& -p_{\chi}\left(k^{\prime} \rho v \frac{\sin \gamma}{\cos ^{2} \gamma} \sin \mu-\frac{v}{r} \sin \gamma \tan L \sin \chi-2 \Omega\left(1+\tan ^{2} \gamma\right) \cos L \cos \chi\right. \\
& \left.+\frac{\Omega^{2} r}{v} \frac{\sin L \cos L \sin \chi \sin \gamma}{\cos ^{2} \gamma}\right)
\end{aligned}
$$

$$
\begin{aligned}
\frac{d p_{L}}{d t}=-p_{v}( & -\Omega^{2} r \sin L(\sin \gamma \cos L-\cos \gamma \sin L \cos \chi) \\
& \left.+\Omega^{2} r \cos L(-\sin \gamma \sin L-\cos \gamma \cos L \cos \chi)\right) \\
-p_{\gamma}( & -2 \Omega \sin L \sin \chi-\frac{\Omega^{2} r}{v} \sin L(\cos \gamma \cos L+\sin \gamma \sin L \cos \chi) \\
& \left.+\frac{\Omega^{2} r}{v} \cos L(-\cos \gamma \sin L+\sin \gamma \cos L \cos \chi)\right) \\
-p_{l} \frac{v}{r} \frac{\cos \gamma \sin \chi \sin L}{\cos ^{2} L} & -p_{\chi}\left(\frac{v}{r} \cos \gamma\left(1+\tan { }^{2} L\right) \sin \chi+2 \Omega(\cos L+\tan \gamma \sin L \cos \chi)\right. \\
& \left.+\frac{\Omega^{2} r}{v} \frac{\cos ^{2} L \sin \chi}{\cos ^{2}}-\frac{\Omega^{2} r}{v} \frac{\sin ^{2} L \sin \chi}{\cos \gamma}\right)
\end{aligned}
$$

$$
\begin{aligned}
\frac{d p_{l}}{d t}= & 0 \\
\frac{d p_{\chi}}{d t}= & -p_{v} \Omega^{2} r \cos L \cos \gamma \sin L \sin \chi-p_{\gamma}\left(2 \Omega \cos L \cos \chi-\frac{\Omega^{2} r}{v} \cos L \sin L \sin \gamma \sin \chi\right) \\
& +p_{L} \frac{v}{r} \cos \gamma \sin \chi-p_{l} \frac{v}{r} \frac{\cos \gamma \cos \chi}{\cos L} \\
& -p_{\chi}\left(\frac{v}{r} \cos \gamma \tan L \cos \chi+2 \Omega \tan \gamma \cos L \sin \chi+\frac{\Omega^{2} r}{v} \frac{\sin L \cos L \cos \chi}{\cos \gamma}\right)
\end{aligned}
$$

For an extremal starting from $q_{0}$ on the initial manifold (see Table 1), and $p_{0}=\left(0,0,-3.10^{-8}, 10^{-8}, 0,0\right)$, we can compute the first conjugate time using the algorithm given previously. This leads to $t_{1 c}=392 \mathrm{~s}$. 
Remark 3.1. The analysis of the extremal flow $^{5}$ is extremely complicated. Indeed there are many singularities because the equations are meromorphic due to the switching surface $p_{\gamma}=p_{\chi}=0$; moreover existence of optimal solutions is not ensured in general because the problem is not convex and there exist singular extremals. This has a consequence on the classification of extremals ${ }^{9}$. Fortunately, in our optimal problem, little is needed about the classification of extremals, because of our boundary conditions and more precisely because $\chi(0), \chi\left(t_{f}\right)$ and $\gamma\left(t_{f}\right)$ are free. Actually the transversality conditions yield the following results.

Lemma 3.1. If the initial or terminal longitude is free, then $p_{l} \equiv 0$ and the problem reduces to control a subsystem $\dot{q}=f(q, \mu), q=(r, v, \gamma, L, \chi) \in \mathbb{R}^{5}$.

Lemma 3.2. If $\Omega=0$ and if the initial condition on $(L, l, \chi)$ is free then $p_{\chi} \equiv p_{L} \equiv$ $p_{l} \equiv 0$ and the problem reduces to control the subsystem $\dot{q}_{1}=X\left(q_{1}\right)+u_{1} Y_{1}\left(q_{1}\right)$, with $q_{1}=(r, v, \gamma)$, describing the longitudinal motion.

This reduction is a key point of our analysis.

\subsection{Constraints and boundary arcs}

Constraints only concern the coordinates $(r, v, \gamma)$, and are of generic order 2 . We give below the details of the computations for the thermal flux and normal acceleration (actually the constraint on the dynamic pressure happens to be not active in our problem, due to the boundary conditions).

The iso-flux phase. This phase of the flight, corresponding to a maximal thermal flux, is characterized by the equations $\varphi=\varphi^{\max }, \dot{\varphi}=0$. Derivating the latter equality yields $\ddot{\varphi}=A+B \cos \mu+C \sin \mu=0$, where the coefficients $A, B, C$, may be calculated using formal computations as

$$
\begin{aligned}
A= & \frac{1}{4} \frac{C_{q} \sqrt{\rho} v}{h_{s}^{2} r^{4}}\left(v^{4} r^{4}-12 h_{s}{ }^{2} \Omega^{4} r^{6} \cos ^{4} L \cos ^{2} \gamma \cos ^{2} \chi-4 v^{3} h_{s} r^{4} \Omega \cos \gamma \cos L \sin \chi\right. \\
& +24 h_{s}^{2} g_{0} \Omega^{2} r^{3} \sin \gamma \cos \gamma \sin L \cos L \cos \chi+12 v^{2} h_{s} r^{5} \Omega^{2} \sin \gamma \cos \gamma \sin L \cos L \cos \chi \\
& -24 \Omega^{2} v^{2} r^{4} h_{s}^{2} \sin \gamma \cos \gamma \sin L \cos L \cos \chi-24 h_{s}{ }^{4} \Omega^{4} r^{6} \sin \gamma \cos \gamma \sin L \cos ^{3} L \cos \chi \\
& -24 g_{0} r^{2} v h_{s}{ }^{2} \Omega \cos \gamma \cos L \sin \chi+24 \Omega^{3} r^{5} v h_{s}{ }^{2} \cos \gamma \cos L \sin \chi+12 h_{s}^{2} \Omega^{4} r^{6} \cos ^{4} L \\
& -12 \Omega^{2} r^{4} v^{2} h_{s}{ }^{2} \cos ^{2} \gamma \cos ^{2} L \cos ^{2} \chi+12 h_{s}^{2} \Omega^{4} r^{6} \cos ^{2} \gamma \cos ^{2} L \cos \chi-14 v^{2} h_{s} r^{5} \Omega^{2} \cos ^{2} L \\
& +24 g_{0} h_{s}{ }^{2} \Omega^{2} r^{3} \cos ^{2} \gamma \cos ^{2} L-12 \Omega^{4} r^{6} h_{s}^{2} \cos ^{2} \gamma \cos ^{4} L-12 \Omega^{2} r^{4} v^{2} h_{s}^{2} \cos ^{2} \gamma \cos ^{2} L \\
& +12 v^{2} \Omega^{2} r^{4} h_{s}^{2} \cos ^{2} L-48 h_{s}{ }^{2} g_{0} \Omega^{2} r^{3} \cos ^{2} L+12 v^{2} h_{s} r^{5} \Omega^{2} \cos ^{2} \gamma \cos ^{2} L \\
& -36 g_{0} h_{s}^{2} v^{2} r \cos ^{2} \gamma-12 v^{2} h_{s} r^{2} g_{0} \cos ^{2} \gamma+12 v^{2} \Omega^{2} r^{4} h_{s}{ }^{2} \cos ^{2} \gamma+12 \Omega^{4} r^{6} h_{s}^{2} \cos ^{2} L \\
& +26 v^{4} h_{s} r^{4} k \rho \sin \gamma+48 h_{s}^{2} k^{2} \rho^{2} v^{4} r^{4}-72 h_{s}^{2} k \rho v^{2} r^{5} \Omega^{2} \sin \gamma \cos ^{2} L \\
& +72 h_{s}^{2} g_{0} k \rho v^{2} r^{2} \sin \gamma+72 h_{s}^{2} k \rho v^{2} r^{5} \Omega^{2} \cos \gamma \sin L \cos L \cos \chi-v^{4} r^{4} \cos ^{2} \gamma \\
& \left.-2 v^{4} h_{s} r^{3} \cos ^{2} \gamma-12 g_{0}{ }^{2} h_{s}^{2} \cos ^{2} \gamma+24 v^{2} g_{0} r h_{s}^{2}+24 h_{s}^{2} g_{0}{ }^{2}+14 v^{2} h_{s} r^{2} g_{0}\right),
\end{aligned}
$$




$$
\begin{gathered}
B=-\frac{1}{4} \frac{C_{q} \sqrt{\rho} v}{h_{s}^{2} r^{4}}\left(2 v^{4} h_{s} r^{4} k^{\prime} \rho \cos \gamma+12 g_{0} h_{s}^{2} k^{\prime} \rho v^{2} r^{2} \cos \gamma-12 \Omega^{2} r^{5} h_{s}^{2} k^{\prime} \rho v^{2} \cos \gamma \cos ^{2} L\right. \\
\left.-12 \Omega^{2} r^{5} h_{s}^{2} k^{\prime} \rho v^{2} \sin \gamma \sin L \cos L \cos \chi\right), \\
C=3 C_{q} k^{\prime} \rho^{3 / 2} v^{3} \Omega^{2} r \sin L \cos L \sin \chi .
\end{gathered}
$$

The term $C$ is related to the Coriolis and centripetal effects and we notice numerically that $B<0$. In particular if $\Omega$ is neglected, $C=0$ and we get $A+B u_{1}=0, u_{1}=\cos \mu$. There is a solution $\left|u_{1}\right| \leq 1$ if $B \neq 0$ and $|A / B| \leq 1$. If $u_{1}$ is not saturating to \pm 1 , it corresponds to two distinct roots $\pm u_{2}$, with $u_{2}=\sin \mu>0$. The effect of $u_{2}$ on the lateral motion is to increase or decrease $\dot{\chi}\left( \pm u_{2}\right)$ with respect to $\dot{\chi}(0)$. More generally if $\Omega$ is not neglected and taking the bank angle in ] $-\pi, \pi]$, the control $\mu$ (if exists), is

$$
\mu=\operatorname{Arcsin}-C / \sqrt{B^{2}+C^{2}} \pm \operatorname{Arccos}-A / \sqrt{B^{2}+C^{2}} \quad[2 \pi] .
$$

Actually the sign + is convenient for numerical reasons. Indeed the sign - would lead $L(t)$ to be decreasing; this would prevent the trajectory from reaching the desired final point $L\left(t_{f}\right)$. In fact we shall see that the latitude $L(t)$ of the complete optimal trajectory is an increasing function of time.

The iso-normal acceleration phase. As before a boundary arc is located on $\gamma_{n}=\gamma_{n}^{\max }, \dot{\gamma}_{n}=0$, and the boundary control is solution of $\ddot{\gamma}_{n}=D+E \cos \mu+$ $F \sin \mu=0$, where

$$
\begin{gathered}
D=\frac{2 \rho}{h_{s}^{2} r^{4}}\left(h_{s}^{2} g_{0}^{2}+v^{4} r^{4}-v^{4} \cos ^{2} \gamma h_{s} r^{3}+6 h_{s}^{2} k^{2} \rho^{2} v^{4} r^{4}+5 v^{2} h_{s} r^{2} g_{0}\right. \\
+4 g_{0} v^{2} r h_{s}^{2}+7 v^{4} \sin \gamma h_{s} r^{4} k \rho+4 v^{2} \sin \gamma h_{s} r^{5} \Omega^{2} \cos \gamma \sin L \cos L \cos \chi \\
-4 v^{2} \cos ^{2} \gamma h_{s} r^{2} g_{0}-2 v^{3} \cos \gamma h_{s} r^{4} \Omega \cos L \sin \chi+4 v^{2} h_{s} r^{5} \Omega^{2} \cos ^{2} \gamma \cos ^{2} L \\
+8 h_{s}^{2} g_{0} k \rho v^{2} r^{2} \sin \gamma-8 h_{s}^{2} k \rho v^{2} r^{5} \Omega^{2} \sin \gamma \cos ^{2} L-6 g_{0} h_{s}^{2} v^{2} r \cos ^{2} \gamma \\
+8 h_{s}^{2} k \rho v^{2} r^{5} \Omega^{2} \cos \gamma \sin L \cos L \cos \chi-4 g_{0} r^{2} v h_{s}^{2} \Omega \cos \gamma \cos L \sin \chi \\
-4 \Omega^{2} v^{2} r^{4} h_{s}{ }^{2} \sin \gamma \cos \gamma \sin L \cos L \cos \chi-2 \Omega^{2} r^{4} v^{2} h_{s}{ }^{2} \cos ^{2} \gamma \cos ^{2} L \\
-2 \Omega^{2} r^{4} h_{s}^{2} \cos ^{2} \gamma \cos ^{2} L v^{2} \cos ^{2} \chi-5 v^{2} h_{s} r^{5} \Omega^{2} \cos ^{2} L \\
-4 h_{s}{ }^{2} g_{0} \Omega^{2} r^{3} \cos ^{2} L+2 \Omega^{2} v^{2} r^{4} h_{s}^{2} \cos ^{2} L-v^{4} r^{4} \cos ^{2} \gamma \\
\left.+2 \Omega^{2} r^{4} h_{s}^{2} v^{2} \cos ^{2} \gamma+4 \Omega^{3} r^{5} h_{s}^{2} v \cos \gamma \sin \chi \cos L+2 \Omega^{4} r^{6} h_{s}^{2} \cos ^{2} L\right) \\
E=-\frac{\rho^{2} k^{\prime} v^{2}}{r^{2} h_{s}}\left(-2 \Omega^{2} r^{3} h_{s} \sin \gamma \sin L \cos L \cos \chi+v^{2} r^{2} \cos \gamma\right. \\
\left.-2 \Omega^{2} r^{3} h_{s} \cos \gamma \cos { }^{2} L+2 g_{0} h_{s} \cos \gamma\right), \\
F=2 k^{\prime} \rho^{2} r v^{2} \Omega^{2} \sin L \cos L \sin \chi .
\end{gathered}
$$




\subsection{Longitudinal motion}

Notice that if the rotation $\Omega$ of the Earth may be neglected, the system governing the longitudinal motion is of the form $\dot{q}=X(q)+u Y(q)$, where $q=(r, v, \gamma)$ and $u=\cos \mu$ with $|u| \leq 1$. The state constraints are of the form $c_{i}(q) \leq 0$ for $i=1,2,3$. The vectors fields $X, Y$ defined by (1.9) are

$$
X=(v \sin \gamma) \psi \frac{\partial}{\partial r}-\left(g \sin \gamma+k \rho v^{2}\right) \psi \frac{\partial}{\partial v}+\cos \gamma\left(-\frac{g}{v}+\frac{v}{r}\right) \psi \frac{\partial}{\partial \gamma}, Y=k^{\prime} \rho v \psi \frac{\partial}{\partial \gamma},
$$

where $k=\frac{1}{2} \frac{S C_{D}}{m}$ and $k^{\prime}=\frac{1}{2} \frac{S C_{L}}{m}$. We now recall the results of ${ }^{3}$ concerning the longitudinal motion.

Lemma 3.3. In the flight domain where $\cos \gamma \neq 0$, we have:

(1) $X, Y,[X, Y]$ are linearly independent.

(2) $[Y,[X, Y]] \in \operatorname{span}\{Y,[X, Y]\}$.

(3) $[X,[X, Y]](q)=a(q) X(q)+b(q) Y(q)+c(q)[X, Y](q)$, where $a<0$.

\section{Proposition 3.1.}

(1) The small time reachable set $R\left(q_{0}\right)$ is homeomorphic to a closed convex cone where the boundary is formed by the surfaces $S_{1}, S_{2}$, where $S_{1}$ is the union of trajectories of the form $q_{-} q_{+}$representing an arc with control $u=-1$ followed by an arc with control $u=+1 ; S_{2}$ is the union of trajectories of the form $q_{+} q_{-}$.

(2) Each point in the interior of the cone is reached by an unique trajectory of the form $q_{-} q_{+} q_{-}$and $q_{+} q_{-} q_{+}$, an arc $q_{-} q_{+} q_{-}$being time minimal and an arc $q_{+} q_{-} q_{+}$being time maximal.

(3) If $T$ is small enough the set of points reached at minimal time $T$ is homeomorphic to a closed disk in the plane, whose boundary is formed by extremities of arcs $q_{-} q_{+}$and $q_{+} q_{-}$and interior of arcs $q_{-} q_{+} q_{-}$, where in the second case each arc of the sequence is not empty.

In order to take into account the state constraints, we need some hypotheses.

Assumptions. The system is of the form $\dot{q}=X(q)+u Y(q)$ where $u=\cos \mu$ and each state constraint is of the form $c(q) \leq 0$. If the constraints are of generic order $m$, the secondary constraints, $\dot{c}=\ldots=c^{(m-1)}=0$ write $Y c=Y X c=Y X^{m-2} c=0$, and a boundary control is computed by solving $c^{(m)}=X^{m} c+u Y X^{m-1} c=0$, where a vector field $Z$ acts on a function $f$ by Lie derivative. Hence a boundary control along a generic boundary arc $q_{b}$ writes $u_{b}=-X^{m} c_{\mid q_{b}} / Y X^{m-1} c_{\mid q_{b}}$.

Let $t \mapsto q_{b}(t), t \in[0, T]$, be a boundary arc associated to the control $u_{b}$. We assume that $\left(C_{1}\right) Y X^{m-1} c_{\mid q_{b}} \neq 0$, where $m$ is the generic order, and $\left(C_{2}\right)\left|u_{b}\right|<$ 1 on $[0, T]$, i.e. the boundary control is admissible and not saturating. From the computations of Section 3.2, we have the following.

Lemma 3.4. The constraints on the thermal flux and on the normal acceleration are of order 2 in the flight domain where $\cos \gamma \neq 0$. Moreover assumption $\left(C_{1}\right)$ holds along any boundary arc. 
Definition 3.1. The order of contact of $\operatorname{arcs} q_{-}, q_{+}$with the boundary arc in $c=\dot{c}=0$ is greater than or equal to 2 , and if assumption $\left(C_{2}\right)$ holds, the order is 2 . In this case both arcs are denoted $q_{-}^{T}, q_{+}^{T}$. A bridge between two constraints is an $\operatorname{arc} q_{-}$or $q_{+}$tangent to both constraints with order 2 , denoted $q_{-}^{B}$ or $q_{+}^{B}$.

Proposition 3.2. Assume $\left(C_{2}\right)$ holds along the boundary arc in the flight domain of the space shuttle. Then a boundary arc is small time optimal, and a small time optimal trajectory is of the form $q_{-} q_{+}^{T} q_{b} q_{+}^{T} q_{-}$.

\subsection{Remarks on bang-bang extremals $(\cos \mu= \pm 1$ )}

The cost extended system is of the form

$$
\dot{q}_{1}=f_{1}\left(q_{1}, \cos \mu\right)+\Omega C(q, \Omega), \dot{q}_{2}=f_{2}(q, \sin \mu)+O(\Omega), \dot{l}=f_{3}(q), \dot{q}_{0}=f_{4}\left(q_{1}\right),
$$

where $q_{1}=(r, v, \gamma), q_{2}=(L, \chi), q=\left(q_{1}, q_{2}\right)$ and $f_{4}\left(q_{1}\right)=C_{q} \sqrt{\rho} v^{3}$. We observe that the control $\mu=k \pi, k=0$ or 1 , is singular, and the linearized system along the corresponding trajectory writes

$$
\begin{aligned}
& \dot{\delta_{1} q_{1}}=\frac{\partial f_{1}}{\partial q_{1}} \delta_{1} q_{1}-\frac{\partial f_{1}}{\partial u_{1}} \sin \mu \delta \mu+\Omega \frac{\partial C}{\partial q_{1}} \delta_{1} q_{1}+\Omega \frac{\partial C}{\partial q_{2}} \delta_{1} q_{2} \\
& \dot{\delta_{1} q_{2}}=\frac{\partial f_{2}}{\partial q} \delta_{1} q+\frac{\partial f_{2}}{\partial u_{2}} \cos \mu \delta \mu+O(\Omega), \dot{\delta_{1}} l=\frac{\partial f_{3}}{\partial q} \delta q, \quad \dot{\delta_{1}} q_{0}=\frac{\partial f_{4}}{\partial q_{1}} \delta q_{1}
\end{aligned}
$$

where $\partial C / \partial q_{2}=0$ for $L=0$ and $\chi= \pm \pi / 2$. Moreover $\sin \mu=0$ implies $\delta_{1} q_{1} \equiv 0$. On the other part

$$
\begin{aligned}
& \dot{\delta_{1} L}=-\frac{v}{r} \cos \gamma \sin \chi \delta_{1} \chi, \dot{\delta_{1}} l=\frac{v}{r} \cos \gamma\left(\frac{\cos \chi}{\cos L} \delta_{1} \chi+\frac{\sin \chi \sin L}{\cos ^{2} L} \delta_{1} L\right), \\
& \dot{\delta_{1} \chi}=\frac{k^{\prime} \rho v}{\cos \gamma} \cos \mu \delta \mu+\frac{v}{r} \cos \gamma\left(\tan L \cos \chi \delta_{1} \chi+\frac{\sin \chi}{\cos ^{2} L} \delta_{1} L\right)+O(\Omega) .
\end{aligned}
$$

If $\Omega$ is neglected, the longitudinal motion is decoupled of the lateral motion. Let $K(t)$ denote the image of $L^{\infty}$ by the Fréchet derivative of the end-point mapping for the extended system.

Lemma 3.5. If $\Omega=0$, the control $\mu=k \pi$ is a constant extremal control of order 0 , and $K(t) \subset \operatorname{span}\left\{\partial / \partial q_{2}, \partial / \partial l\right\}$. Let $a=v \cos \gamma \sin \chi \sin L / r \cos ^{2} L$, $b=-\operatorname{cotan} \chi / \cos L$. If $\chi \neq k \pi$ and $a \neq \dot{b}$, the linearized system (3.3) is controllable.

Proof. One has $\partial / \partial \chi \in K(t)$, and the controllability of (3.3) is equivalent to the controllability of the 2-dimensional system

$$
\dot{\delta_{1} L}=-\frac{v}{r} \cos \gamma \sin \chi \delta_{1} \chi, \dot{\delta_{1} l}=\frac{v}{r} \cos \gamma\left(\frac{\cos \chi}{\cos L} \delta_{1} \chi+\frac{\sin \chi \sin L}{\cos ^{2} L} \delta_{1} L\right),
$$

where $\delta_{1} \chi$ can be considered as a control. If $\sin \chi \neq 0$, up to the feedback transformation $u(t)=-\frac{v}{r} \cos \gamma \sin \chi \delta_{1} \chi$, the system reduces to $\dot{\delta_{1}} L=u(t)$, 
$\dot{\delta_{1}} l=a(t) \delta_{1} L+b(t) u(t)$ and can be written in the form $\dot{x}=A(t) x+B(t) u$. The system is not controllable if and only if there exists $p \neq 0$ such that $\dot{p}=-p A$ and $p B=0$. Differentiating we get $p(-A B+\dot{B})=0$. This yields the sufficient condition $a \neq \dot{b}$. This condition, if satisfied for each $t$, is the regularity condition of ${ }^{4}$ to compute a normal form in the generic case.

\subsection{Preliminary observation: a particular extremal}

Here, there is no need to neglect $\Omega$. We observe that trajectories such that $\chi(0)=$ $\pm \pi / 2$ and $L(0)=0$ with control $\sin \mu=0$ satisfy $\chi(t)= \pm \pi / 2$ and $L(t)=0$ for each $t$. Moreover $\partial C / \partial q_{2}=0$ if $\chi= \pm \pi / 2$ and $L=0$ and hence $\delta_{1} q_{1}(t)=0$ if $\delta_{1} q_{1}(0)=0$. Finally from our previous computations $K(t)=\operatorname{span}\left\{\partial / \partial q_{2}\right\}$ and $\delta_{1} l(t)=0$. Hence

Lemma 3.6. Even if the Earth rotation is not neglected, if $\chi(0)= \pm \pi / 2$ and $L(0)=0$, then bank angles $\mu=k \pi$ are constant extremal controls such that $\chi(t)=$ $\pm \pi / 2, L(t)=0$ and $K(t)=\operatorname{span}\left\{\partial / \partial q_{2}\right\}$. Such trajectories are extremals of the accessory problem of extremizing $l\left(t_{f}\right)$ where the transfer time $t_{f}$ is fixed.

We next analyze the reachable set near the previous extremal. We assume $\chi(0)=$ $\pi / 2$, the case $\chi(0)=-\pi / 2$ being similar (and not needed).

Proposition 3.3. In the flight domain, trajectories with initial conditions $\chi(0)=$ $\pi / 2, L(0)=0$ and corresponding to controls $\cos \mu= \pm 1$ are maximizing $l\left(t_{f}\right), t_{f}$ fixed, for the $L^{\infty}$-topology on the set of controls.

Proof. Computing near $\chi(0)=\pi / 2, L(0)=0$, one has

$$
\begin{aligned}
& \dot{\delta_{2} l}=\frac{v}{r} \cos \gamma\left(\frac{\delta_{1} L^{2}}{2}-\frac{\delta_{1} \chi^{2}}{2}\right), \quad \dot{\delta_{1}} L=\frac{v}{r} \cos \gamma\left(-\delta_{1} \chi\right), \\
& \dot{\delta_{1} \chi} \quad=\frac{k^{\prime} \rho v \cos \mu}{\cos \gamma} \delta \mu+\frac{v}{r} \cos \gamma \delta_{1} L+O(\Omega)
\end{aligned}
$$

This system describes the projection on the space $(L, l, \chi)$ of the reachable set for the $L^{\infty}$-topology near the reference trajectory so that $\cos \mu= \pm 1$ and $\sin \mu=0$. Introducing the parameterization $d \sigma=\frac{v}{r} \cos \gamma d t$ the system (3.5) can be written as

$$
\frac{d \delta_{2} l}{d \sigma}=\frac{1}{2}\left(\delta_{1} L^{2}-\delta_{1} \chi^{2}\right), \frac{d \delta_{1} L}{d \sigma}=-\delta_{1} \chi, \frac{d \delta_{1} \chi}{d \sigma}=\alpha \delta_{1} L+\beta \delta u,
$$

where $\beta$ is not vanishing. The quadratic form $\delta_{2} l\left(\sigma_{f}\right)=\int_{0}^{\sigma_{f}} \frac{1}{2}\left(\delta_{1} L^{2}-\delta_{1} \chi^{2}\right) d \sigma$ together with the boundary conditions $\delta_{1} L=\delta_{1} \chi=0$ at times 0 and $\sigma_{f}$, represents the intrinsic second-order derivative in the direction $p_{l} \neq 0$, the other components of $p$ being 0 . To conclude we need the following lemma.

Lemma 3.7. ${ }^{4,21}$ The quadratic form $\delta_{2} l\left(\sigma_{f}\right)$ with $\delta_{1}^{\cdot} L=-\delta_{1} \chi, \delta_{1} \dot{\chi} \chi=v$ and the boundary conditions $\delta_{1} L(0)=\delta_{1} L\left(\sigma_{f}\right)=0$ is negative up to a first conjugate time $t_{1 c}$ given by $\pi=\int_{0}^{t_{1 c}} \frac{v}{r} \cos \gamma d t$. 
A rough numerical estimate gives $t_{1 c}>2500 s$. The flight duration being less than $1300 s$, the proposition follows.

Remark 3.2. Notice that in our evaluation of the conjugate time, the smoothness of the reference extremal is not required. Hence our proposition still holds for a concatenation of bang arcs $q_{-}$and $q_{+}$corresponding to $\cos \mu= \pm 1$.

Our preliminary analysis permits to construct an approximation of the optimal policy. The trajectory splits into two parts.

\subsection{Joining the iso-flux phase, and Coriolis effect}

The boundary values $r(0), v(0), \gamma(0), L(0), r\left(t_{f}\right), v\left(t_{f}\right), L\left(t_{f}\right), l\left(t_{f}\right)$ are fixed, $l(0)$ is fixed or free and $\chi(0), \gamma\left(t_{f}\right), \chi\left(t_{f}\right)$ are free, see Table 1 .

Lemma 3.8. If $\Omega=0$, then for all control $\mu$, the trajectory starting from $(r(0), v(0), \gamma(0))$ corresponding to $\mu$, violates the constraint on the thermal flux before reaching the desired terminal point.

Proof. Assume $\Omega=0$ and consider the longitudinal motion. According to proposition 3.1, the small time reachable set is bounded by surfaces $S_{1}, S_{2}$ formed by $\operatorname{arcs} q_{+} q_{-}$and $q_{-} q_{+}$. Hence it is sufficient to check that all trajectories of type $q_{+} q_{-}$ and $q_{-} q_{+}$starting from the initial point violate the constraint on the flux, which is easy to check numerically.

As a consequence, at the beginning of the trajectory the rotation $\Omega$ of the earth cannot be neglected. This can be easily understood if we consider in (1.1) the equation $\dot{\gamma}=\left(-\frac{g}{v}+\frac{v}{r}\right) \cos \gamma+k^{\prime} \rho v \cos \mu+F_{c}+F_{e}$, where $F_{c}=2 \Omega \cos L \sin \chi$ is the Coriolis component, and $F_{e}=\Omega^{2} \frac{r}{v} \cos L(\cos \gamma \cos L+\sin \gamma \sin L \cos \chi)$ is the centripetal component. At the entrance in the atmosphere, the lift force is small and $F_{c}$ has to be used to compensate the gravitational term $-g / v$. In particular, at the beginning we must have $F_{c}+F_{e}>0$. Concretely the Coriolis force helps to lift up the nose of the shuttle so that it does not violate the constraint on the thermal flux.

Lemma 3.9. At the beginning of the flight $F_{e}=o\left(F_{c}\right), F_{e}+F_{c} \sim F_{c}$ and $F_{c}$ has its maximal value for $L=0$ and $\chi=\pi / 2$.

Since $L(0)=0$, we are led to choose $\chi(0)$ near $\pi / 2$. Actually one can check on numerical simulations that trajectories which do not violate the constraint on the thermal flux have indeed their initial azimuth $\chi(0) \approx \pi / 2$.

Proposition 3.4. If we assume $\Omega=0$ and $l(0)$ free, by modifying slightly the initial data $r(0), v(0)$ and $\gamma(0)$ of Table 1 , the optimal policy of the whole system is the optimal policy of the projected 3-dimensional system controlled by $u_{1}=\cos \mu$ and 
describing the longitudinal motion, $L$ being increasing and $L\left(t_{f}\right)$ is adjusted using $\chi(0)$.

Proof. We choose $q_{1}(0)=(r(0), v(0), \gamma(0))$ closed enough from the initial data such that the Coriolis effect is not needed and the reachable set meet the constraint on the thermal flux where the boundary control is admissible. Consider after reparemetrization by the flux, the associated optimal control problem. For the longitudinal motion the terminal manifold $M_{1}$ given by $r\left(t_{f}\right), v\left(t_{f}\right)$ fixed, $\gamma\left(t_{f}\right)$ free and let $t_{f}^{*}$ be the optimal time. Then the optimal time $t_{f}^{* *}$ for the whole system satisfies $t_{f}^{* *} \geq t_{f}^{*}$. Hence assume we can find $\chi(0)$ such that $L\left(t_{f}^{*}\right)$ has the desired value, then it is the optimal policy.

In our problem the boundary conditions on the latitude are $L(0)=0, L\left(t_{f}\right)=$ $10.99 \mathrm{deg}$ and from our previous analysis $\chi(0)$ is near $\pi / 2$. The control $u_{1}=\cos \mu$ is defined by the longitudinal motion and $u_{2}=\sin \mu$ is chosen such that $\chi \in[0, \pi / 2]$ and hence $L$ increases. This amounts to choose, along the constraint one of the two boundary described in section 3.2. For instance, for the constraint on the thermal flux we choose the root such that $\sin \mu<0$ and $\chi$ decreases from $\chi(0) \approx \pi / 2$.

\subsection{The second part of the trajectory}

Actually the assumption $\Omega \neq 0$ is crucial only when tracking the constraint on the thermal flux and we assume from now on that $\Omega=0$. Indeed it may be checked numerically that, from this point, the Coriolis and centripetal forces may be neglected with respect to friction and gravitational forces. We have two problems to analyze: the initial longitude can be free or fixed. The first case is simpler and analyzed next.

Problem 1: the initial longitude is free. Since the variable $l$ does not appear in the dynamics, our problem reduces to a 5-dimensional system in the variables $(r, v, \gamma, L, \chi)$. Knowing $l\left(t_{f}\right)$, the convenient initial longitude $l(0)$ is computed by backward integration.

It can be checked numerically using the boundary conditions that both constraints on the thermal flux and on the normal acceleration are active but if one saturates the constraint on the dynamic pressure, the desired final point is not accessible. Hence this latter constraint is not active in our problem.

Proposition 3.5. ${ }^{3}$ Consider the system $\dot{q}=X+u Y, q \in \mathbb{R}^{3},|\mathrm{u}| \leq 1$, describing the longitudinal motion with the two constraints $c_{i}(q) \leq 0, i=1,2$ on the thermal flux and the normal acceleration. The small time optimal policy is of the form $q_{-} q_{+}^{T} q_{\text {flux }} q_{+}^{B} q_{a c c} q_{+}^{T} q_{-}$, where $q_{\text {flux }}$ (resp. $q_{a c c}$ ) is a boundary arc corresponding to the constraint on the thermal flux (resp. on the normal acceleration), and $q_{+}^{B}$ is the bridge between the constraints on the thermal flux and on the normal acceleration.

The previous proposition describes locally the optimal policy for the longitudinal motion with fixed boundary conditions on $(r, v, \gamma)$. In our problem the terminal path 
inclination $\gamma$ is free, and a straightforward computation shows that the optimal policy is of the form $q_{-} q_{+}^{T} q_{f l u x} q_{+}^{B} q_{a c c} q_{+}^{T}$, a switching being removed at the end because $p_{\gamma}\left(t_{f}\right)=0$ (and thus the final arc $q_{-}$is trivial). Using the remarks of the previous section, this policy actually holds for the whole system, $L\left(t_{f}\right)$ being adjusted with $\chi(0)$. To make this result global (in the sense of the transfer time), we use numerical simulations based on the following facts. Global optimal results are related to the topological properties of the mapping $E_{1}: q_{0} \mapsto q_{-} q_{+}^{T} q_{f l u x} q_{+}^{B} q_{a c c} q_{+}^{T}\left(q_{0}\right)$, which describes the extremal flow along the reference trajectory. They can be investigated either theoretically or numerically by checking that the above policy is the only extremal policy.

Remark 3.3. We assume $\Omega=0$ in the second part of the trajectory. The approximation $\sin \mu \approx 0$ outside the constraints can be checked by inspecting the extremal flow, showing that $p_{\chi} \ll p_{\gamma}$ except on a short duration in the first part before reaching the constraint and in the second part when starting from the constraint. Numerical simulations are given on Fig. 2.

Fig. 2. The extremal flow.

The peaks of the ratio $p_{\chi} / p_{\gamma}$ observed on the figures are due to the vanishing of $p_{\gamma}$, and in our approximation policy they correspond to switching times. Outside these peaks the ratio $\left|p_{\chi} / p_{\gamma}\right|$ is uniformly very small, less than $10^{-3}$. Hence the following proposition holds with a very good approximation.

Proposition 3.6. For the space shuttle re-entry problem where the initial longitude is not fixed, the optimal policy corresponding to the boundary conditions is, in approximation, of the form $q_{-} q_{+}^{T} q_{\text {flux }} q_{+}^{B} q_{a c c} q_{+}^{T}$.

Problem 2: the initial longitude is fixed. We have the following result.

Proposition 3.7. For the space shuttle re-entry problem where the initial longitude 
is fixed, the optimal policy corresponding to the boundary conditions is, in approximation, of the form $q_{-} q_{+}^{T} q_{f l u x} q_{+}^{T} q_{-}$.

Proof. Our proof is related to numerical simulations. In the first problem where the initial longitude is free, numerical simulations yield $l\left(t_{f}\right)-l(0) \simeq 40 \mathrm{deg}$; in the second problem the value imposed by the CNES is about $50 \mathrm{deg}$. Hence, in the second problem, the strategy consists in increasing the final longitude. Notice that $i=v \cos \gamma \sin \chi / r \cos L$, where $L$ is close to 0 , and moreover numerically we check that $\chi(t) \in(0, \pi / 2]$ necessarily; hence $l$ is an increasing function of $t$. In particular, in the second part of the trajectory, we can reparametrize the system by the longitude $l$, and if $\Omega$ is neglected the system writes

$$
\begin{aligned}
\frac{d r}{d l}=r \frac{\tan \gamma \cos L}{\sin \chi}, & \frac{d v}{d l}=-k \rho r \frac{v}{\cos \gamma} \frac{\cos L}{\sin \chi}-\frac{g r \cos L \tan \gamma}{v \sin \chi}, \\
\frac{d L}{d l}=\frac{\cos L}{\tan \chi}, & \frac{d \chi}{d l}=\frac{k^{\prime} \rho}{\cos ^{2} \gamma} \frac{\cos L}{\sin \chi} \sin \mu+\sin L .
\end{aligned}
$$

In particular, if $\sin \mu=0$, we have $\tan \chi \tan L d L=d \chi$. Therefore our control problem reduces to reach the desired value $r\left(l_{f}\right), v\left(l_{f}\right)$ and $L\left(l_{f}\right)$ where $l_{f}$ is fixed, whereas $\chi(0)$ is used to adjust $L\left(l_{f}\right)$. Hence a final $\operatorname{arc} q_{+}^{T} q_{-}$is needed to reach the terminal point. On the other hand, a numerical computation shows that in order to increase the final longitude we must decrease the time along the boundary of the domain and in particular we cannot saturate the constraint on the normal acceleration.

As in problem 1, our result is only an approximation of the optimal policy. Indeed, outside the constraints, the approximation $p_{\chi} \ll p_{\gamma}$ holds except during a short duration and similarly to the previous problem it can be checked numerically using the extremal flow.

On the other part, the global aspect of the policy is as previously a consequence of the topological properties of the surface $E_{2}\left(q_{0}\right)=q_{-} q_{+}^{T} q_{\text {flux }} q_{+}^{T} q_{-}\left(q_{0}\right)$ with respect to the terminal manifold where $q_{0}$ belongs to the initial manifold defined by the initial conditions.

\section{Numerical simulations}

Having reduced the problem to determine an approximation of the optimal policy among a concatenation of bang and boundary arcs, either $q_{-} q_{+}^{T} q_{f l u x} q_{+}^{B} q_{a c c} q_{+}^{T}$ or $q_{-} q_{+}^{T} q_{f l u x} q_{+}^{T} q_{-}$, the switchings times are computed using a standard multipleshooting algorithm ${ }^{19,6,11}$. The algorithm is written in Fortran and simulations were led using Matlab ${ }^{\mathrm{b}}$.

Our reduction procedure avoids the implementation of the complete extremal system. This is essential in order to improve the convergence of the algorithm. The results are the following, we distinguish between both problems.

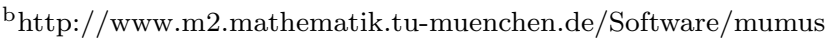


Problem 1: initial longitude not fixed. Switching times and initial values of longitude and azimuth have to be determined by the multiple-shooting method. More precisely, the first switching time, from $q_{-}$to $q_{+}$, allows to adjust the entry in the iso-flux phase, which is characterized by $\varphi=\varphi^{\max }, \dot{\varphi}=0$; the third switching time, from $q_{\text {flux }}$ to $q_{+}$, is used to adjust the entry in the iso-normal acceleration phase; the fifth switching time, from $q_{\text {acc }}$ to $q_{+}$, permits to adjust the final velocity $v\left(t_{f}\right)$; the initial azimuth $\chi(0)$ is used to adjust the terminal latitude $L\left(t_{f}\right)$. On the other part the final time is determined by the final altitude. Results are drawn on Fig. 3, Fig. 4 and Fig. 5.

Fig. 3. State coordinates in problem 1.

Problem 2: initial longitude fixed. Switching times and the initial value of azimuth have to be determined by the multi-shooting method. More precisely, the first switching time, from $q_{-}$to $q_{+}$, allows to adjust the entry in the iso-flux phase; the third switching time, from $q_{\text {flux }}$ to $q_{+}$, permits to adjust the final velocity $v\left(t_{f}\right)$; the fourth switching time, from $q_{+}$to $q_{-}$, is used to adjust the final longitude $l\left(t_{f}\right)$; the initial azimuth $\chi(0)$ allows to adjust the terminal latitude $L\left(t_{f}\right)$. Results are drawn on Fig. 6, Fig. 7 and Fig. 8.

\section{Appendix: numerical data}

- Earth radius $r_{T}=6378139 \mathrm{~m}$; gravity model: $g(r)=\frac{g_{0}}{r^{2}}$, with $g_{0}=3.9800047 .10^{14} \mathrm{~m}^{3} \cdot \mathrm{s}^{-2}$; 
Fig. 4. Bank angle in problem 1.

Fig. 5. State constraints in problem 1.

Earth rotation velocity $\Omega=7.292115853608596 .10^{-5}$ rad. $\mathrm{s}^{-1}$.

- Atmospheric density $\rho(r)=\rho_{0} \exp \left(-\left(r-r_{T}\right) / h_{s}\right)$, with $\rho_{0}=1.225 \mathrm{~kg} . \mathrm{m}^{-3}$ and $h_{s}=7143 \mathrm{~m}$.

- Sound velocity $v_{\mathrm{Son}}(r)=\sum_{i=0}^{5} a_{i} r^{i}$, where

$$
\begin{aligned}
& a_{5}=-1.880235969632294 .10^{-22}, \quad a_{4}=6.074073670669046 .10^{-15}, \\
& a_{3}=-7.848681398343154 .10^{-8}, \quad a_{2}=5.070751841994340 .10^{-1}, \\
& a_{1}=-1.637974278710277 .10^{6}, \quad a_{0}=2.116366606415128 .10^{12} .
\end{aligned}
$$


Fig. 6. State coordinates in problem 2.

Fig. 7. Bank angle in problem 2.

- Mach number $\operatorname{Mach}(v, r)=v / v_{\mathrm{Son}}(r)$.

- Shuttle data: mass $m=7169.602 \mathrm{~kg}$; reference surface $S=15.05 \mathrm{~m}^{2}$;

drag coefficient $k=S C_{D} / 2 m$;

lift coefficient $k^{\prime}=S C_{L} / 2 m$;

- Aerodynamic coefficients:

Table of $C_{D}($ Mach, incidence $)$ 
Fig. 8. State constraints in problem 2.

\begin{tabular}{r|lllllllllll} 
& 0.00 & 10.00 & 15.00 & 20.00 & 25.00 & 30.00 & 35.00 & 40.00 & 45.00 & 50.00 & $55.00 \mathrm{deg}$ \\
\hline 0.00 & 0.231 & 0.231 & 0.269 & 0.326 & 0.404 & 0.500 & 0.613 & 0.738 & 0.868 & 0.994 & 1.245 \\
2.00 & 0.231 & 0.231 & 0.269 & 0.326 & 0.404 & 0.500 & 0.613 & 0.738 & 0.868 & 0.994 & 1.245 \\
2.30 & 0.199 & 0.199 & 0.236 & 0.292 & 0.366 & 0.458 & 0.566 & 0.688 & 0.818 & 0.948 & 1.220 \\
2.96 & 0.159 & 0.159 & 0.195 & 0.248 & 0.318 & 0.405 & 0.509 & 0.628 & 0.757 & 0.892 & 1.019 \\
3.95 & 0.133 & 0.133 & 0.169 & 0.220 & 0.288 & 0.373 & 0.475 & 0.592 & 0.721 & 0.857 & 0.990 \\
4.62 & 0.125 & 0.125 & 0.160 & 0.211 & 0.279 & 0.363 & 0.465 & 0.581 & 0.710 & 0.846 & 0.981 \\
10.00 & 0.105 & 0.105 & 0.148 & 0.200 & 0.269 & 0.355 & 0.458 & 0.576 & 0.704 & 0.838 & 0.968 \\
20.00 & 0.101 & 0.101 & 0.144 & 0.205 & 0.275 & 0.363 & 0.467 & 0.586 & 0.714 & 0.846 & 0.970 \\
30.00 & 0.101 & 0.101 & 0.444 & 0.208 & 0.278 & 0.367 & 0.472 & 0.591 & 0.719 & 0.849 & 0.972 \\
50.00 & 0.101 & 0.101 & 0.144 & 0.208 & 0.278 & 0.367 & 0.472 & 0.591 & 0.719 & 0.849 & 0.972 \\
Mach & & & & & & & & & & &
\end{tabular}

Table of $C_{L}($ Mach, incidence $)$

\begin{tabular}{r|lllllllllll}
0.00 & 0.00 & 10.00 & 15.00 & 20.00 & 25.00 & 30.00 & 35.00 & 40.00 & 45.00 & 50.00 & $55.00 \mathrm{deg}$ \\
\hline 0.00 & 0.000 & 0.185 & 0.291 & 0.394 & 0.491 & 0.578 & 0.649 & 0.700 & 0.729 & 0.734 & 0.756 \\
2.00 & 0.000 & 0.185 & 0.291 & 0.394 & 0.491 & 0.578 & 0.649 & 0.700 & 0.729 & 0.734 & 0.756 \\
2.30 & 0.000 & 0.172 & 0.269 & 0.363 & 0.454 & 0.535 & 0.604 & 0.657 & 0.689 & 0.698 & 0.723 \\
2.96 & 0.000 & 0.154 & 0.238 & 0.322 & 0.404 & 0.481 & 0.549 & 0.603 & 0.639 & 0.655 & 0.649 \\
3.95 & 0.000 & 0.139 & 0.215 & 0.292 & 0.370 & 0.445 & 0.513 & 0.569 & 0.609 & 0.628 & 0.626 \\
4.62 & 0.000 & 0.133 & 0.206 & 0.281 & 0.358 & 0.433 & 0.502 & 0.559 & 0.600 & 0.620 & 0.618 \\
10.00 & 0.000 & 0.103 & 0.184 & 0.259 & 0.337 & 0.414 & 0.487 & 0.547 & 0.591 & 0.612 & 0.609 \\
20.00 & 0.000 & 0.091 & 0.172 & 0.257 & 0.336 & 0.416 & 0.490 & 0.552 & 0.596 & 0.616 & 0.612 \\
30.00 & 0.000 & 0.087 & 0.169 & 0.258 & 0.338 & 0.418 & 0.493 & 0.555 & 0.598 & 0.619 & 0.613 \\
50.00 & 0.000 & 0.087 & 0.169 & 0.258 & 0.338 & 0.418 & 0.493 & 0.555 & 0.598 & 0.619 & 0.613 \\
Mach & & & & & & & & & & &
\end{tabular}

- Incidence profile imposed: if the Mach number is larger than 10 then the incidence is set to 40. If the Mach number is between 2 and 10 then the incidence is a linear function of the Mach number between the values 12 and 40 . If the Mach number is less than 2 then the incidence is set to 12 (see Fig. 9).

- State constraints:

Constraint on the thermal flux $\varphi=C_{q} \sqrt{\rho} v^{3} \leq \varphi^{\max }$, where $C_{q}=1.705 .10^{-4}$ S.I. and $\varphi^{\max }=717300 \mathrm{~W} \cdot \mathrm{m}^{-2}$. 
Fig. 9. Incidence profile in function of the Mach number

Constraint on the normal acceleration $\gamma_{n}=\frac{S}{2 m} \rho v^{2} \sqrt{C_{D}^{2}+C_{L}^{2}} \leq \gamma_{n}^{\max }$, where $\gamma_{n}^{\max }=29.34 \mathrm{~m} \cdot \mathrm{s}^{-2}$.

Constraint on the dynamic pressure $P=\rho v^{2} / 2 \leq P^{\max }=25000 \mathrm{kPa}$.

\section{Appendix 2: numerical simulations without taking into account the state constraints}

In this appendix we give the numerical results for the problem of steering the shuttle from the initial to the final set prescribed by Table 1, minimizing the total thermal flux, but without taking into account the state constraints. In this case an approximation of the optimal policy is a concatenation of bang arcs, namely $q_{-} q_{+}$ in the first problem (initial longitude not fixed) or $q_{-} q_{+} q_{-}$in the second problem (initial longitude fixed).

Problem 1: initial longitude not fixed. The switching time and initial values of longitude and azimuth are determined by the multiple-shooting method. More precisely the (unique) switching time permits to adjust the final velocity $v\left(t_{f}\right)$, and the initial azimuth $\chi(0)$ is used to adjust the terminal latitude $L\left(t_{f}\right)$. Results are drawn on Fig. 10, Fig. 11 and Fig. 12.

Problem 2: initial longitude fixed. The switching times and the initial value of azimuth are determined by the multiple-shooting method. More precisely the first (resp. second) switching time permits to adjust the final velocity $v\left(t_{f}\right)$ (resp. the final longitude $l\left(t_{f}\right)$ ), and the initial azimuth $\chi(0)$ is used to adjust the terminal latitude $L\left(t_{f}\right)$. Results are drawn on Fig. 13, Fig. 14 and Fig. 15

In both cases, state constraints are indeed violated, as expected.

\section{References}

1. A. Agrachev and A. Sarychev, On abnormal extremals for Lagrange variational problems, J. Math. Syst. Estim. Cont. 81 (1998) 87-118.

2. B. Bonnard and M. Chyba, The role of singular trajectories in control theory, 
Fig. 10. State coordinates in problem 1.

Fig. 11. Bank angle in problem 1.

Mathématiques \& Applications 40 (Springer-Verlag, 2003).

3. B. Bonnard, L. Faubourg, G. Launay and E. Trélat, Optimal control with state constraints, and the space shuttle re-entry problem, J. Dyn. Cont. Syst. 92 (2003) 155199.

4. B. Bonnard and I. Kupka, Théorie des singularités de l'application entrée/sortie et optimalité des trajectoires singulières dans le problème du temps minimal, Forum Mathematicum 52 (1993) 111-159.

5. B. Bonnard and E. Trélat, Une approche géométrique du contrôle optimal de l'arc atmosphérique de la navette spatiale, ESAIM Cont. Optim. Calc. Var. 7 (2002) 179222 . 
Fig. 12. State constraints in problem 1.

Fig. 13. State coordinates in problem 2.

6. R. Bulirsch, Die Mehrzielmethode zur numerischen Lösung von nichtlinearen Randwertproblemen und Aufgaben der optimalen Steuerung, DLR, Oberpfaffenhofen (1971). 
Fig. 14. Bank angle in problem 2.

Fig. 15. State constraints in problem 2.

7. CNES, Mécanique spatiale (Cepadues Eds, 1993).

8. J. M. Coron and L. Praly, Guidage en rentrée atmosphérique, Rapport 415 CNES (2000).

9. I. Ekeland, Discontinuité des champs Hamiltoniens et existence des solutions optimales en calcul des variations, Publications IHES 47 (1977) 5-32.

10. J. Harpold and C. Graves, Shuttle entry guidance, J. Astr. Sci. 27 (1979) 239-268.

11. P. Hiltmann, Numerische Lösung von Mehrpunkt-Randwertproblemen und Aufgaben der optimalen Steuerung mit Steuerfunktionen über endlichdimensionalen Räumen, Dissertation, Univ. München (1990). 
12. A. Ioffe and V. Tikhomirov, Theory of extremal problems (North-Holland Publishing, 1979).

13. D. Jacobson, M. Lele and J. L. Speyer, New necessary conditions of optimality for control problems with state-variable inequality constraints, J. Math. Analysis Appl. 35 (1971) 255-284.

14. A. J. Krener and H. Shättler, The structure of small-time reachable sets in low dimensions, SIAM J. Cont. Optim. 271 (1989) 120-147.

15. H. Maurer, On optimal control problems with bounded state variables and control appearing linearly, SIAM J. Cont. Optim. 153 (1977) 345-362.

16. A. Miele, Recent advances in the optimization and guidance of aeroassociated orbital transfers, Acta Astr. 3810 (1996) 747-768.

17. L. S. Pontryagin, V. G. Boltyanskij, R. V. Gamkrelidze and E. F. Mishchenko, The mathematical theory of optimal processes (John Wiley \& Sons, 1962).

18. A. Sarychev, The index of the second variation of a control system, Math. USSR Sbornik 413 (1982) 338-401.

19. J. Stoer and R. Bulirsch, Introduction to numerical analysis (Springer-Verlag, 1980).

20. H. J. Sussmann, Envelopes, high-order optimality conditions and Lie brackets, Proceedings of the 28th IEEE Conference on Decision and Control 1-3 (1989) 1107-1112.

21. E. Trélat, Optimality of singular trajectories and asymptotics of accessibility sets under generic assumptions, Contemporary Trends in Nonlinear Geometric Control Theory and its Applications (World Scientific, 2002). 